\title{
Geometric shaping: low-density coding of Gaussian-like constellations
}

\author{
Joseph J. Boutros*, Uri Erez ${ }^{\dagger}$, Johannes Van Wonterghem ${ }^{\ddagger}$, Gil I. Shamir ${ }^{\S}$, and Gilles Zémor \\ *Texas A\&M University, Qatar, boutros@tamu.edu. ${ }^{\dagger}$ Tel Aviv University, Israel, uri@eng.tau.ac.il. \\ ${ }^{\ddagger}$ Ghent University, Belgium, johannes.vanwonterghem@ugent.be. ${ }^{\S}$ Google Pittsburgh, USA, gshamir@ieee.org. \\ 『Bordeaux University, France, gilles.zemor@math.u-bordeaux.fr.
}

\begin{abstract}
Constellation shaping is necessary to approach channel capacity for information rates above $1 \mathrm{bit} / \mathrm{dim}$. Probabilistic shaping shows a small gap to capacity, however a complex distribution matcher is required to modify the source distribution. Spherical shaping of lattice constellations also reduces the gap to capacity, but practical Voronoi shaping is feasible in small dimensions only. In this paper, our codebook is a real geometrically nonuniform Gaussian-like constellation. We prove that this discrete codebook achieves channel capacity when the number of points goes to infinity. Then we build a special mapping to interface between non-binary low-density codes and the codebook, allowing the code alphabet size to be equal to the square root of the codebook size. Excellent performance is shown with fast-encoding and practical iterative probabilistic decoding, e.g. $0.7 \mathrm{~dB}$ gap to capacity at $6 \mathrm{bits} / \mathrm{s} / \mathrm{Hz}$ with a code defined over the ring $\mathbb{Z} / 8 \mathbb{Z}$.
\end{abstract}

\section{INTRODUCTION}

When targeting high transmission rates, it is necessary to incorporate shaping with high-order modulations. Many different methods to attain shaping have been proposed over the years, the unifying element among them is that of leveraging codebooks that have a uniform (marginal) distribution over a finite alphabet and then applying some operation to alter the distribution to be more Gaussian-like. Examples include applying a distribution matching mapping as proposed by Gallager in a quite general context [1], cutting a spherical region out of the full cubic lattice. Alternatively one can replace the spherical shaping region with a lattice Voronoi region which may be achieved via the technique of trellis shaping [2]. As a practical embodiment of the latter approach, one may take a coding lattice that allows for efficient iterative decoding as envisaged in [3] and brought to fruition in [4].

It is also possible to directly shape via the direct sum of a low-dimensional sub-lattice to get significant shaping gain that adds up to the coding gain such as Leech-shaped LDA constellations [5] reaching a gap to capacity of $0.8 \mathrm{~dB}$ with 2.7 bits/dim. Such performance is also attained via probabilistic shaping where the sign bit of a constellation carries uniformlydistributed parity bits [6]. The amplitude of probabilisticshaped constellations is usually forced to follow a MaxwellBoltzmann distribution, as suggested in [7], via the application of a distribution matcher [8] [9] to the source symbols. Our motivation in designing the real Gaussian-like codebook in this paper is to find a simple alternative to both lattice shaping and distribution matching.

Our approach for building a capacity-approaching codebook is similar to the construction initiated by Sun and van Tilborg in [10]. They proposed a non-uniform constellation on the real line with a uniform probability distribution. Sun and van Tilborg's codebook is obtained by two steps: Equal probability partitioning followed by a centroid computation. Our codebook is obtained in one step as described in the next section. Sun and van Tilborg's approach was brought to the complex plane by Méric [11]. Another recent approach based on tiling triangles makes a circular bi-dimensional constellation for both geometric and probabilistic shaping [12].

The present work describes a practical embodiment of the geometrically non-uniform approach where the underlying code is a non-binary ( $q$-ary) low-density parity-check (LDPC) code and the mapping is to the real line. As a rationale for choosing this method, we note that it is known that the ensemble average spectrum of regular $q$-ary LDPC codes, for $q$ prime, approaches that of an i.i.d. uniform code as the right and left degrees of the tanner graph grow. This result was first established in [13] (see also [3]) and was re-derived and extended in [14], [15] where shaping and iterative decoding were also applied. In particular the latter work provides a useful benchmark for comparison of the results presented in the present work; see Section V.

\section{DEFINITION OF A MONO-DIMENSIONAL DISCRETE GAUSSIAN CODEBOOK}

Let $p$ be a positive integer, $p \geq 2$. The Gaussian codebook $\mathcal{G}$ is a finite discrete set of $p$ points on the real line, $\mathcal{G}=$ $\left\{x_{0}, x_{1}, \ldots, x_{p-1}\right\}$, where $x_{i} \in \mathbb{R}$ and $x_{i}<x_{i+1}$. The ring $\mathbb{Z} / p \mathbb{Z}$ of integers modulo $p$ will be denoted by $\mathbb{Z}_{p}$. The discrete set $\mathcal{U}=\left\{u_{0}, u_{1}, \ldots, u_{p-1}\right\}$ has $p$ equidistant points in the interval $[0,1]$ given by

$$
u_{i}=\frac{1}{2 p}+\frac{i}{p}, \quad i=0 \ldots p-1 .
$$

Let $s \in \mathbb{Z}_{p}=\{0,1,2, \ldots, p-1\}$ be a symbol from a uniform $p$-ary information source. The symbol $s$ is mapped into a point $x(s)$ in $\mathcal{G}$ as follows:

- Map from $\mathbb{Z}_{p}$ to $\mathcal{U}$ :

$$
u(s)=\frac{1}{2 p}+\frac{(s+\lfloor p / 2\rfloor) \bmod p}{p},
$$

where $\lfloor x\rfloor$ is the greatest integer less than or equal to $x$. 
- Map from $\mathcal{U}$ to the real line:

$$
x(s)=\phi^{-1}(u(s)), \text { or equivalently } x_{i}=\phi^{-1}\left(u_{i}\right),
$$

for $i=(s+\lfloor p / 2\rfloor) \bmod p$, where $\phi^{-1}(u)$ is the inverse function of $\phi(x)$,

$$
\phi(x)=\int_{-\infty}^{x} f(t) d t, \quad f(x)=\frac{1}{\sqrt{2 \pi}} e^{-\frac{x^{2}}{2}} .
$$

$\phi(x)$ is the well-known distribution function of a zero-mean unit-variance real Gaussian random variable. An illustration of $\mathcal{G}$ is given in Figure 1 for different values of the constellation size $p$. Note that for odd values of $p, s=0$ is mapped to $x(0)=x_{(p-1) / 2}=0$ whereas for even $p, s=0$ and $p-1$ are mapped to $\phi^{-1}\left(\frac{1}{2} \pm \frac{1}{2 p}\right)$. In both cases (even and odd $p$ ) no points are mapped to infinity. We also define $x_{\max }$ as the maximal Euclidean distance between the constellation $\mathcal{G}$ and the origin,

$$
x_{\max }=\left|x_{0}\right|=x_{p-1},
$$

and $x_{\text {min }}$ as the minimal Euclidean distance between $\mathcal{G} \backslash\{0\}$ and the origin,

$$
x_{\text {min }}=\left|x_{\lfloor p / 2\rfloor-1}\right|=x_{\lfloor(p+1) / 2\rfloor} .
$$
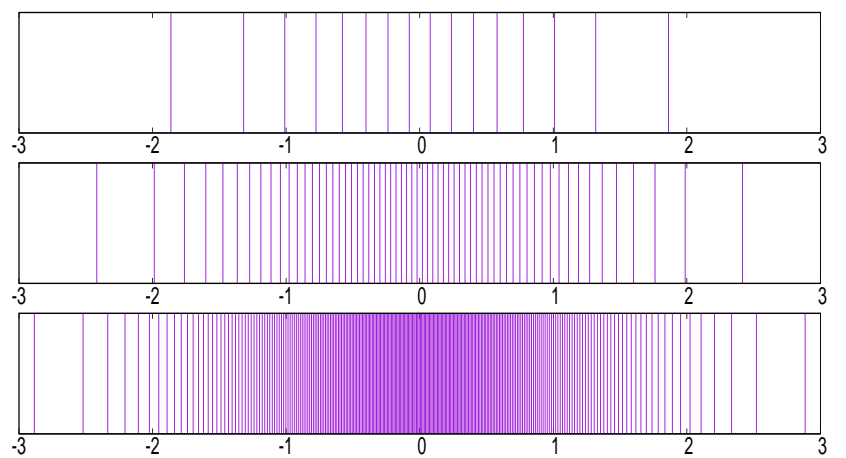

Fig. 1. Spectrogram-like illustration of the $p$ points of the discrete Gaussian codebook $\mathcal{G}$ for $p=16, p=64$, and $p=256$ from top to bottom.

\section{Distance, Energy, And Mutual Information}

Let us determine the principal characteristics of the discrete codebook $\mathcal{G}$ in terms of minimum distance, average energy, and peak to average power ratio. We also prove that channel capacity is achieved for $p \rightarrow \infty$ at the end of this section.

Proposition 1: The minimum Euclidean distance of the discrete Gaussian codebook $\mathcal{G}$ satisfies

$$
d_{\text {min }}(\mathcal{G})=\frac{\sqrt{2 \pi}}{p}+o\left(\frac{1}{p^{2}}\right) .
$$

Proof: Let $\kappa=1$ for odd $p$ and $\kappa=2$ for even $p$, where $p \geq 2$. Using the smallest non-zero amplitude $x_{\min }$ in $\mathcal{G}$, we have $d_{\text {min }}=\kappa x_{\text {min }}$. But $x_{\text {min }}$ is solved from

$$
x_{\text {min }}=\phi^{-1}\left(\frac{1}{2}+\frac{1}{\kappa p}\right)
$$

The Taylor series of $\phi^{-1}(u)$ around $u=1 / 2$ reduces to $x_{\min }=\frac{\sqrt{2 \pi}}{\kappa p}+o\left(\frac{1}{p^{2}}\right)$, where the second derivative is zero. Finally, $d_{\min }=\kappa x_{\min }$ yields the announced result.

Proposition 2: The largest amplitude $x_{\max }$ in the constellation $\mathcal{G}$, defined by (5), satisfies

$$
x_{\max } \leq \sqrt{2 \log (p)} .
$$

Proof: The largest amplitude can be solved from

$$
x_{\max }=\phi^{-1}\left(1-\frac{1}{2 p}\right) .
$$

for any $p \geq 2$. Then $\phi\left(x_{\max }\right)=1-\frac{1}{2 p}=1-Q\left(x_{\max }\right)$, where $Q(x)$ is the Gaussian tail function. Finally, $\frac{1}{2 p}=Q\left(x_{\max }\right) \leq$ $\frac{1}{2} e^{-\frac{x_{\max }^{2}}{2}}$ gives the announced result.

Now we consider the average energy $E_{s}$ of $\mathcal{G}$. All points are equiprobable since we assume a uniform $p$-ary source.

Proposition 3: For any $p=|\mathcal{G}| \geq 2$, the second moment of the constellation $\mathcal{G}=\phi^{-1}(\mathcal{U})$ satisfies

$$
E_{s}(\mathcal{G})=\frac{\sum_{i=0}^{p-1} x_{i}^{2}}{p}<1 .
$$

Further, for $p \rightarrow \infty, E_{s}(\mathcal{G}) \rightarrow 1$.

Proof: The function $\phi^{-1}(u)^{2}$ is integrable

$$
\int_{0}^{1} \phi^{-1}(u)^{2} d u=\int_{-\infty}^{\infty} x^{2} f(x) d x=1 .
$$

The second derivative of $\phi^{-1}(u)^{2}$ is shown to be

$$
\left(\phi^{-1}(u)^{2}\right)^{\prime \prime}=\frac{2\left(1+x^{2}\right)}{f(x)^{2}}>0,
$$

i.e. $\phi^{-1}(u)^{2}$ is concave up. Now, call $\Delta_{i}$ the sub-interval of length $\left|\Delta_{i}\right|=1 / p$ centered on $u_{i}$ and apply Jensen's inequality

$$
\frac{1}{\left|\Delta_{i}\right|} \int_{\Delta_{i}} \phi^{-1}(u)^{2} d u>\phi^{-1}\left(\int_{\Delta_{i}} d u\right)^{2}
$$

to get

$$
\int_{u_{i}-1 / 2 p}^{u_{i}+1 / 2 p} \phi^{-1}(u)^{2} d u>\frac{\phi^{-1}\left(u_{i}\right)^{2}}{p} .
$$

After summing all integrals over the $p$ sub-intervals, we obtain

$$
M_{p}=\frac{\sum_{i=0}^{p-1} \phi^{-1}\left(u_{i}\right)^{2}}{p}<\int_{0}^{1} \phi^{-1}(u)^{2} d u,
$$

which becomes via (12)

$$
M_{p}=\frac{\sum_{i=0}^{p-1} x_{i}^{2}}{p}=E_{s}(\mathcal{G})<1 .
$$

The second part of this lemma is proven by redefining the sub-interval $\Delta_{i}=\left[u_{i-1}, u_{i}\right]$, i.e. moving the point $u_{i}$ from the middle to the right. Consider the sum

$$
\begin{aligned}
R_{p} & =2 \sum_{i=1}^{\lfloor p / 2\rfloor-1} \frac{\phi^{-1}\left(u_{i}\right)^{2}}{p} \\
& =2 \int_{0}^{\frac{1}{2}} \sum_{i=1}^{\lfloor p / 2\rfloor-1} \phi^{-1}\left(u_{i}\right)^{2} W_{p}\left(u-\frac{i}{p}\right) d u
\end{aligned}
$$


where the indicator function $W_{p}(u)$ is 1 in the window $\left[\frac{-1}{2 p}, \frac{1}{2 p}\right]$ and 0 outside. The sequence of functions $\sum_{i=1}^{[p / 2]-1} \phi^{-1}\left(u_{i}\right)^{2} W_{p}\left(u-\frac{i}{p}\right)$ converges pointwise to $\phi^{-1}(u)^{2}$ which is also monotone decreasing in the interval $[0,1 / 2]$, so the sequence of functions is also dominated by $\phi^{-1}(u)^{2}$. By Lebesgue's dominated convergence theorem we get the limit

$$
\lim _{p \rightarrow \infty} R_{p}=2 \int_{0}^{\frac{1}{2}} \phi^{-1}(u)^{2} d u=1 .
$$

On the other hand, $R_{p}=E_{s}(\mathcal{G})-2 \frac{x_{0}^{2}}{p}$. But from (5) and Proposition $2, \frac{x_{0}^{2}}{p}=\frac{x_{\max }^{2}}{p} \leq \frac{2 \log p}{p}$ vanishes with $p$. Finally, we have $E_{s} \rightarrow R_{p} \rightarrow 1$ when $p \rightarrow \infty$.

More elaborated algebra proves that $E_{s}(\mathcal{G}) \geq 1-O\left(\frac{\log p}{p}\right)$.

The mapping from $\mathcal{U}$ to $\mathbb{R}$ makes the non-uniform Gaussianlike geometric distribution of $\mathcal{G}$ to have higher density around the origin and less density at the tails. As the constellation size $p$ grows, the discrete induced non-uniform constellation approaches the Gaussian one in the following sense, where $X_{p}$ and $U_{p}$ are two random variables.

Lemma 1: For any $p$, let $X_{p}=\phi^{-1}\left(U_{p}\right)$ be the induced discrete constellation where $U_{p}$ is uniform over the discrete set $\mathcal{U}$ defined in (1). Then the random variable $X_{p} \rightarrow \mathcal{N}(0,1)$ in distribution as $p \rightarrow \infty$.

Proof: We follow in the footsteps of Hugo Méric [11]. Denote by

$$
\psi(\omega)=\int_{-\infty}^{\infty} e^{i \omega x} f(x) d x=\int_{0}^{1} e^{i \omega \phi^{-1}(u)} d u
$$

the characteristic function of a standard Gaussian random variable. Further denote the characteristic function of $X_{p}$ by

$$
\psi_{p}(\omega)=\frac{1}{p} \sum_{u_{i}} e^{i \omega \phi^{-1}\left(u_{i}\right)}
$$

Now since the function $e^{i \omega \phi^{-1}(u)}$ is continuous over the interval $[0,1]$, it follows that the Riemann sum (15) converges pointwise to the limit (14). That is, for every $\omega$

$$
\lim _{p \rightarrow \infty} \psi_{p}(\omega)=\psi(\omega)
$$

Thus, by Lévy's theorem, the cumulative distribution function of $X_{p}$ converges to that of the standard normal distribution, i.e., we have weak convergence.

Combining Proposition 3 and Lemma 1, we have by the conditions of Schwarte [16] that

Corollary 1: Given a positive power $P$ and its corresponding constellation scaling factor $\sqrt{P}$, let $X_{p}$ be uniform over the constellation $\sqrt{P} \cdot \mathcal{G}=\sqrt{P} \cdot \phi^{-1}(\mathcal{U})$ and let $\eta \sim \mathcal{N}\left(0, \sigma^{2}\right)$ be an additive white Gaussian noise. Then, the mutual information $I\left(X_{p} ; X_{p}+\eta\right)$ satisfies

$$
\lim _{p \rightarrow \infty} I\left(X_{p} ; X_{p}+\eta\right)=\frac{1}{2} \log \left(1+\frac{P}{\sigma^{2}}\right)
$$

and further $\lim _{p \rightarrow \infty} E\left[X_{p}^{2}\right]=P$.
In presence of a rate- $R_{c}=K / N$ error-correcting code that encodes the source symbols before codebook mapping, the transmitted information rate is $R=R_{c} \times \log _{2}(p)$ bits per dimension, or equivalently $2 R$ bits/s/Hz. The standard signalto-noise ratio (SNR) per bit is defined as $E_{b} / N_{0}=\frac{1}{R} \times \frac{P}{2 \sigma^{2}}$ and $E_{b} / N_{0}(d B)=10 \log _{10}\left(E_{b} / N_{0}\right)$ in decibels. The mutual information $I\left(X_{p} ; X_{p}+\eta\right)$ can now be written as $I_{\mathcal{G}}\left(E_{b} / N_{0}\right)$ and its inverse function as $E_{b} / N_{0}^{\mathcal{G}}(I)$.

Let a $p$-ASK constellation be defined by the mapping $x(s)=-(p-1)+2 s$ on the real line [17]. The coding rate $R_{c}=I_{\mathcal{G}}\left(E_{b} / N_{0}\right) / \log _{2}(p)$ should be selected such that the shaping gain, given by difference $E_{b} / N_{0}^{A S K}(I)(d B)-$ $E_{b} / N_{0}^{\mathcal{G}}(I)(d B)$, is maximized. Numerical plots of $I_{\mathcal{G}}\left(E_{b} / N_{0}\right)$ tell us that the optimal choice is very close to $R_{c}=1 / 2$. Numerical examples at half-rate are found in Table I.

Table I. Shaping gain and gap to capacity for $\mathcal{G}$ with $R_{c}=\frac{1}{2}$.

\begin{tabular}{|r|ccccc|}
\hline Codebook size $p$ & 16 & 32 & 64 & 128 & 256 \\
\hline Shaping gain $(\mathrm{dB})$ & 0.583 & 0.815 & 0.998 & 1.136 & 1.241 \\
\hline Gap to capacity $(\mathrm{dB})$ & 0.182 & 0.136 & 0.104 & 0.083 & 0.064 \\
\hline
\end{tabular}

\section{Codebook MAPPING AND UnCODED ERRoR RATE}

Given the coding rate $R_{c}=\frac{1}{2}$ selected for the rest of this paper, two encoding-mapping methods are possible:

1) $p$-ary code and $p$-ary mapping. In this case, the symbols of the $\mathbb{Z}_{p}$ source are encoded by an error-correcting code defined over $\mathbb{Z}_{p}$ then mapped into a point of $\mathcal{G}$ via $x(s)=\phi^{-1}(u(s)$, $s \in \mathbb{Z}_{p}$ as in (3). The main drawback of this method is the high decoding complexity, since large values of $p$ are required to approach capacity. The main advantage is the direct transfer of soft information from the channel output to the input of a probabilistic LDPC decoder, without any loss.

2) $q$-ary code and $q$-ary mapping, where $q=\sqrt{p}$. Assume that $p$ is a square and let $p=q^{2}$. We can write $s=s_{1} q+s_{2}$, where $s_{1}, s_{2} \in \mathbb{Z}_{q}$. Because $K=N-K$ (half-rate code), we assign most significant positions (i.e. $s_{1}$ ) to the $K$ information symbols and the least significant positions (i.e. $s_{2}$ ) to the $K$ parity symbols. The main drawback of this method is the possible loss of information when converting channel soft information into two soft information for $s_{1}$ and $s_{2}$. However, the iterative probabilistic LDPC decoder allows the generation of extrinsic information that can be used in the detection process between the channel and the decoder. This is wellknown as turbo detection and decoding. Obviously, the great advantage of this second method is an LDPC code defined over $\mathbb{Z}_{\sqrt{p}}$ instead of $\mathbb{Z}_{p}$. Belief propagation on the LDPC Tanner graph becomes much faster, e.g. $q^{2}$ transitions in the syndrome trellis representing a check node instead of $p^{2}$. If a field structure replaces the ring structure, a fast transform of $q \log q$ is much appreciable than $p \log p$ [18] [19].

The two mappings are illustrated in Figure 2. The $p$-ary mapping is in black color on the top. The $q$-ary mapping is below the constellation, in red for the information symbol $s_{1}$ and in blue for the parity symbol $s_{2}$. For the sake of symmetry 
around the origin, the definition of $u(s)$ in the $q$-ary mapping becomes, for $s=s_{1} q+s_{2} \in \mathbb{Z}_{p}$ :

$$
u(s)=\frac{1}{2 p}+\frac{(s+\lfloor(p-q) / 2\rfloor) \bmod p}{p} .
$$
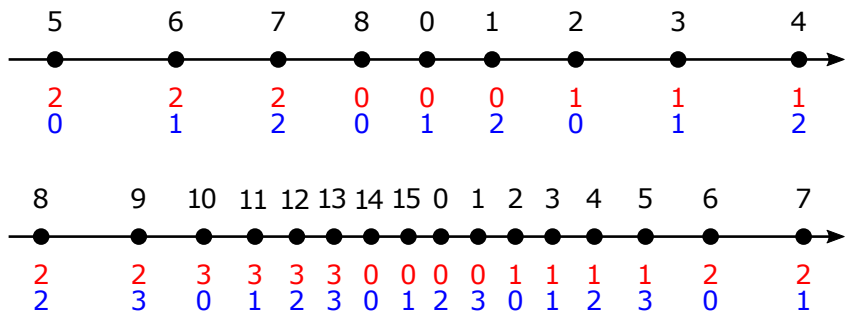

Fig. 2. Codebook examples with $p$-ary and $q$-ary mappings, for two different sizes $p=9(q=3)$ and $p=16(q=4)$.

Now we analyze the uncoded performance on an additive white Gaussian noise (AWGN) channel. Define $d(i, j)$ as the distance between the $i$ th and $j$ th constellation points

$$
d(i, j)= \begin{cases}\left|x_{i}-x_{j}\right| & i, j \in\{0, p-1\} \\ \infty & \text { elsewhere }\end{cases}
$$

The probability of error per symbol for $p$-ary mapping, without coding, is $\operatorname{Pr}_{e, s}=\operatorname{Pr}(\tilde{s} \neq s)$, where $s$ is the transmitted symbol and $\tilde{s}$ is the detected symbol based on the nearest point rule (symbol-by-symbol maximum-likelihood detection). Similarly, let $\mathbf{P r}_{e, s_{1}}=\operatorname{Pr}\left(\tilde{s}_{1} \neq s_{1}\right)$ and $\mathbf{P r}_{e, s_{2}}=\operatorname{Pr}\left(\tilde{s}_{2} \neq\right.$ $s_{2}$ ) be the probability of error per information symbol and the probability of error per parity symbol respectively, for an uncoded codebook with $q$-ary mapping. Then, the two following propositions are proven by applying basic notions from Communication Theory [17],

Proposition 4: For a $p$-ary mapping, the probability of error per symbol is

$$
\mathbf{P r}_{e, s}=\frac{2}{p} \sum_{i=0}^{p-2} Q\left(\frac{d(i, i+1)}{2 \sigma}\right)
$$

Proposition 5: For a $q$-ary mapping, the probability of error per parity symbol is

$$
\begin{aligned}
\operatorname{Pr}_{e, s_{2}} & =\frac{1}{p} \sum_{i=0}^{p-1}\left[\sum _ { j = 1 } ^ { \lceil i / q \rceil } \left(Q\left(\frac{\frac{d(i-j q-1, i-j q)}{2}+d(i-j q, i)}{\sigma}\right)\right.\right. \\
& \left.-Q\left(\frac{\frac{d(i-j q, i-j q+1)}{2}+d(i-j q+1, i)}{\sigma}\right)\right) \\
& +Q\left(\frac{d(i-1, i)}{2 \sigma}\right)+Q\left(\frac{d(i, i+1)}{2 \sigma}\right) \\
& -\sum_{j=1}^{q-\lceil i / q\rceil-1}\left(Q\left(\frac{d(i, i+j q-1)+\frac{d(i+j q-1, i+j q)}{2}}{\sigma}\right)\right. \\
& \left.-Q\left(\frac{\left.d(i, i+j q)+\frac{d(i, i+j q+1)}{2}\right)}{\sigma}\right)\right)
\end{aligned}
$$

The probability of error per information symbol is, for $q$ odd:

$$
\begin{aligned}
& \operatorname{Pr}_{e, s_{1}}=\frac{1}{p} \sum_{i=0}^{p-1}\left[Q\left(\frac{\frac{d(q\lfloor i / q\rfloor-1, q\lfloor i / q\rfloor)}{2}+d(q\lfloor i / q\rfloor, i)}{\sigma}\right)\right. \\
& \left.+Q\left(\frac{d(i, q\lfloor i / q\rfloor+(q-1))+\frac{d(q\lfloor i / q\rfloor+(q-1), q\lfloor i / q\rfloor+q}{2}}{\sigma}\right)\right]
\end{aligned}
$$

For $q$ even, $\mathbf{P r}_{e, s_{1}}$ is given by

$\frac{1}{p}\left[\sum_{i=0}^{p-1-q}\left[Q\left(\frac{\frac{d\left(q\lfloor i / q\rfloor-1+\frac{q}{2}, q\lfloor i / q\rfloor+\frac{q}{2}\right)}{2}+d\left(q\lfloor i / q\rfloor+\frac{q}{2}, i+\frac{q}{2}\right)}{\sigma}\right)\right.\right.$

$+Q\left(\frac{d\left(i+\frac{q}{2}, q\lfloor i / q\rfloor+(q-1)+\frac{q}{2}\right)}{\sigma}+\right.$

$\left.\left.\frac{\frac{d\left(q\lfloor i / q\rfloor+(q-1)+\frac{q}{2}, q\lfloor i / q\rfloor+q+\frac{q}{2}\right)}{2}}{\sigma}\right)\right]$

$+\sum_{i=0}^{\frac{q}{2}-1} Q\left(\frac{d\left(i, \frac{q}{2}-1\right)+\frac{d\left(\frac{q}{2}-1, \frac{q}{2}\right)}{2}}{\sigma}\right)+$

$\left.\sum_{i=p-\frac{q}{2}}^{p-1} Q\left(\frac{\frac{d\left(p-\frac{q}{2}-1, p-\frac{q}{2}\right)}{2}+d\left(p-\frac{q}{2}, i\right)}{\sigma}\right)\right]$

When $\sigma^{2} \ll 1$, at high signal-to-noise ratio, the performance on the AWGN channel is dictated by the figure of merit $d_{\text {min }}^{2} / E_{s}$. For $p$ large enough, the ratio of these figures of merit for $p$-ASK and $\mathcal{G}$ is

$$
\left(\frac{4}{\left(p^{2}-1\right) / 3}\right) /\left(\frac{2 \pi / p^{2}}{1}\right) \approx \frac{6}{\pi}=2.81 \mathrm{~dB} .
$$

In other words, without coding, our discrete Gaussian-like codebook shall exhibit a loss of $2.81 \mathrm{~dB}$ at high SNR with respect to standard ASK modulation! Fortunately, in presence of a strong code, only the low SNR range does matter. From Propositions $4 \& 5$, we illustrate in Table II how $\mathcal{G}$ is beating the $p$-ASK in the low SNR regime, near the capacity limit.

Table II. Probability of error for the uncoded codebook of size $p=256$.

\begin{tabular}{|c|rcc|rcc|}
\hline Gap (dB) to & \multicolumn{3}{|c|}{ Codebook $\mathcal{G}$} & \multicolumn{3}{c|}{$p$-ASK } \\
Capacity & $\mathbf{P r}_{e, s}$ & $\mathbf{P r}_{e, s_{1}}$ & $\mathbf{P r}_{e, s_{2}}$ & $\mathbf{P r}_{e, s}$ & $\mathbf{P r}_{e, s_{1}}$ & $\mathbf{P r}_{e, s_{2}}$ \\
\hline 0.00 & 0.867 & 0.224 & 0.865 & 0.910 & 0.229 & 0.909 \\
0.50 & 0.861 & 0.211 & 0.859 & 0.905 & 0.216 & 0.905 \\
1.00 & 0.854 & 0.200 & 0.853 & 0.899 & 0.204 & 0.899 \\
\hline
\end{tabular}

\section{PERFormanCE WITH NON-BINARY LDPC CODING}

The real discrete Gaussian-like codebook $\mathcal{G}$ with $p=q^{2}$ points is encoded via a regular half-rate LDPC code over the ring $\mathbb{Z}_{q}$ according to the second method described in the previous section. In the Tanner graph of the LDPC code, all $N$ variable nodes (except for the last one) have a degree $d_{v}=2$ and all $N-K=K$ check nodes (except for the first one) have a degree $d_{c}=4$. The adjacency matrix of the Tanner graph has the form $H_{2}=(A \mid B)$, where $A$ is a square $K \times K$ pseudo-random sparse matrix of weight 2 per column and per row. The $K \times K$ matrix $B=\left[b_{i, j}\right]$ is dual diagonal, $b_{i, i}=1, b_{i+1, i}=1$, and the remaining entries are 0 . The final $H$ matrix of the code, defining both the edges and the 


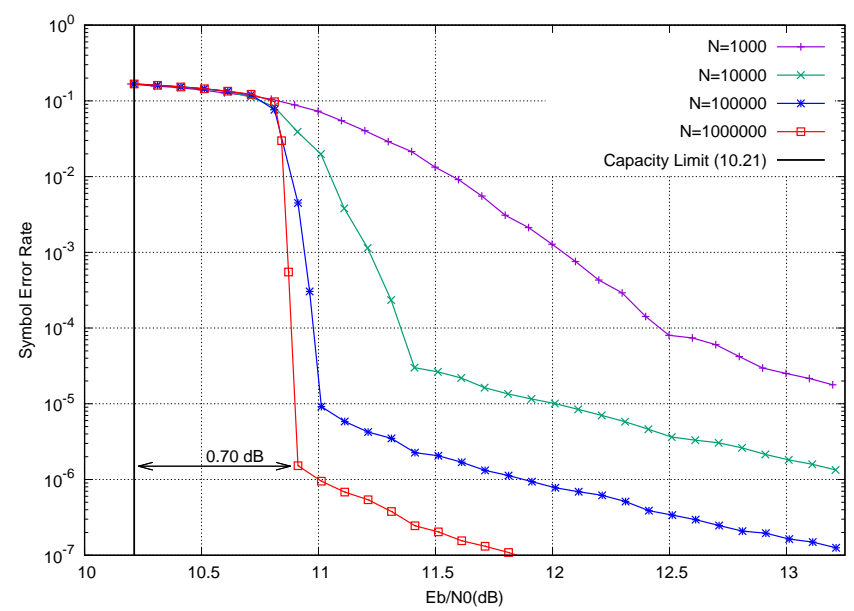

Fig. 3. Symbol error probability versus signal-to-noise ratio for the discrete Gaussian codebook $\mathcal{G}$ of size $p=64$ at different 8 -ary code length $N$.

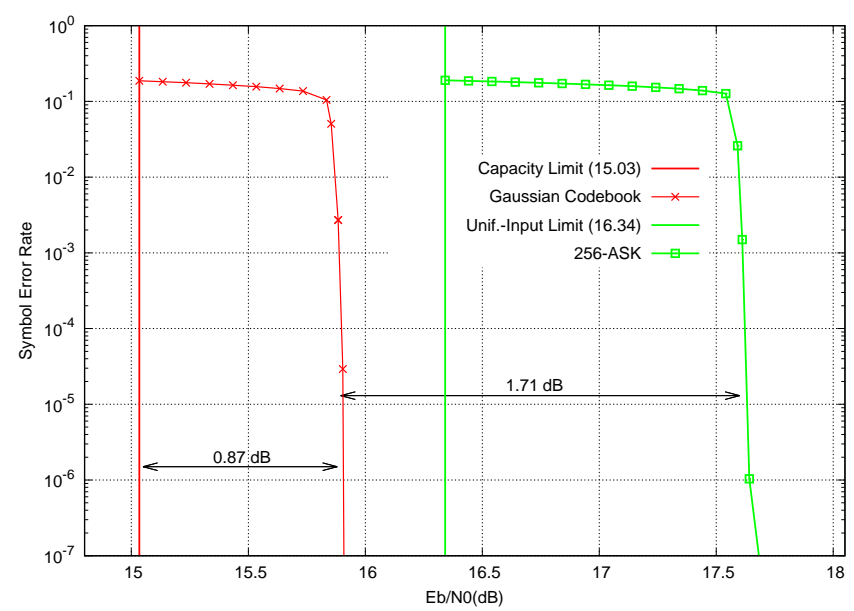

Fig. 4. Gaussian codebook $\mathcal{G}$ of size $p=256$ versus 256-ASK, both with identical 16-ary LDPC coding of length $N=1000000$.

labels of the Tanner graph, is obtained from the binary matrix $\mathrm{H}_{2}$ by replacing each entry equal to 1 by a unit of the ring $\mathbb{Z}_{q}$. Due to lack of space, we cannot say more about the code structure. Briefly, our $(2,4)$-regular LDPC code is a turbo-like repeat-accumulate code over $\mathbb{Z}_{q}$. Encoding is similar to Turbo codes. Decoding can be made via belief propagation along the edges of its Tanner graph.

The symbol error rate (SER) of information symbols versus $E_{b} / N_{0}$, for $p=64$, is plotted in Figure 3 for different values of the code length. The LDPC code is defined over $\mathbb{Z}_{8}$. About 125 decoding iterations were performed (per codeword) in order to reach a stable SER. In our Monte Carlo simulation, we measured at least 100 codeword errors and 500 symbol errors per plotted point. In this scenario, the information rate is $R=3 \mathrm{bits} / \mathrm{dim}$, equivalent to $6 \mathrm{bits} / \mathrm{s} / \mathrm{Hz}$, with a gap to capacity equal to $0.70 \mathrm{~dB}$ at SER in the range $10^{-5} \ldots 10^{-6}$ for the longest code. The total gain at $N=1$ million, with respect to $64-\mathrm{ASK}$, is $1.35 \mathrm{~dB}$ in the same range of SER. Under similar conditions, SER versus $E_{b} / N_{0}$ is plotted in
Figure 4 for $p=256$. With a rate- $\frac{1}{2}$ LDPC code defined over $\mathbb{Z}_{16}$ and a real codebook $\mathcal{G}$ of size $p=256$ points, the gap to capacity is $0.87 \mathrm{~dB}$ (at $8 \mathrm{bits} / \mathrm{s} / \mathrm{Hz}$ !) and the total gain is $1.71 \mathrm{~dB}$ with respect to a 256 -ASK constellation.

\section{Conclusions}

We proposed a simple alternative to probabilistic and to lattice Voronoi shapings. We built a discrete Gaussian-like codebook by mapping $p$ equidistant points in $[0,1]$ to the real line via the inverse distribution function of a normal distribution. Half-rate turbo-like LDPC codes defined over the ring $\mathbb{Z} / q \mathbb{Z}$, where $q=\sqrt{p}$, allow the Gaussian-like codebook to operate near capacity at high spectral efficiency.

\section{REFERENCES}

[1] R. G. Gallager, Information theory and reliable communication, John Wiley and Sons, 1968.

[2] G. D. Forney, "Trellis shaping," IEEE Trans. Inf. Theory, vol. 38, no. 2, pp. 281-300, Mar. 1992.

[3] U. Erez, "Coding with known interference and some results of lattices for digital communication," Ph.D. dissertation, Tel-Aviv University, 2002. [Online] Available: http://www.eng.tau.ac.il/ uri/theses/erez_phd.pdf

[4] N. di Pietro, G. Zémor, and J. J. Boutros, "LDA lattices without dithering achieve capacity on the Gaussian channel," IEEE Trans. Inf. Theory, vol. 64, no. 3, pp. 1561-1594, Mar. 2018.

[5] N. di Pietro and J. J. Boutros, "Leech constellations of ConstructionA lattices," IEEE Trans. Commun., vol. 65, no. 11, pp. 4622-4631, Nov. 2017.

[6] G. Böcherer, F. Steiner, and P. Schulte, "Bandwidth efficient and ratematched low-density parity-check coded modulation," IEEE Trans. Commun., vol. 63, no. 12, pp. 4651-4665, Dec. 2015.

[7] F. R. Kschischang and S. Pasupathy, "Optimal nonuniform signaling for Gaussian channels," IEEE Trans. Inf. Theory, vol. 39, no. 3, pp. 913-929, May 1993.

[8] Z. Wang, A. Patapoutian, R.C. Venkataramani, and G.I. Shamir, "Universal modulation coding for a data channel," US Patent number 8976474 filed in October 2013, Seagate Technology LLC, March 2015.

[9] P. Schulte and G. Böcherer, "Constant composition distribution matching," IEEE Trans. Inf. Theory, vol. 62, no. 1, pp. 430-434, Jan. 2016.

[10] F-W. Sun and H. C. A. van Tilborg, "Approaching capacity by equiprobable signaling on the Gaussian channel," IEEE Trans. Inf. Theory, vol. 39, no. 5, pp. 1714-1716, Sep. 1993.

[11] H. Méric, "Approaching the Gaussian channel capacity with APSK constellations," IEEE Communication Lettersvol. 19, no. 7, pp. 11251128, May 2015.

[12] J.J. Boutros, F. Jardel, and C. Méasson, "Probabilistic shaping and nonbinary codes," in Proc. IEEE International Symp. on Inf. Theory (ISIT), Aachen, June 2017.

[13] U. Erez and G. Miller, "The ML decoding performance of LDPC ensembles over $\mathcal{Z}_{q}$," IEEE Trans. Inf. Theory, vol. 51, no. 5, pp. 18711879, May 2005.

[14] A. Bennatan and D. Burshtein, "On the application of LDPC codes to arbitrary discrete-memoryless channels," IEEE Trans. Inf. Theory, vol. 50, no. 3, pp. 417-438, Mar. 2004.

[15] A. Bennatan and D. Burshtein, "Design and analysis of nonbinary LDPC codes for arbitrary discrete-memoryless channels," IEEE Trans. Inf. Theory, vol. 52, no. 2, pp. 549-583, Feb. 2006.

[16] H. Schwarte, "Approaching capacity of a continuous channel by discrete input distributions," IEEE Trans. Inf. Theoryvol. 42, no. 2, pp. 671675, Mar. 1996.

[17] J. G. Proakis and M. Salehi, Digital communications, 5th ed., New York (NY), USA: McGraw-Hill, 2008.

[18] C. R. P. Hartmann and L. D. Rudolph, "An optimum symbol-by-symbol decoding rule for linear codes," IEEE Trans. on Inf. Theory, 22(5) pp.514517, Sep. 1976.

[19] J. Boutros, A. Ghaith, and Y. Yuan-Wu, "Non-binary adaptive LDPC codes for frequency selective channels: code construction and iterative decoding," in Proc. IEEE Inf. Theory Workshop, pp. 184-188, Chengdu, Oct. 2006. 